\title{
A Comprehensive Method for Quality Evaluation of Houttuyniae Herba by a Single Standard to Determine Multi-components, Fingerprint and HPTLC Method
}

\author{
Wenjing DAI, $*, * *$ Lihua HU,* Lifang JI,* Jing LI,* Kaishun BI,*** and Qing LI*,**† \\ *School of Pharmacy, Shenyang Pharmaceutical University, Shenyang 110016, P. R. China \\ **National and Local United Engineering Laboratory for Key Technology of Chinese Material Medica Quality \\ Control, Shenyang Pharmaceutical University, Shenyang 110016, P. R. China
}

\begin{abstract}
A method of quality evaluation by a single standard to determine multi-components (SSDMC), fingerprint and highperformance thin layer-chromatography (HPTLC) was developed for traditional Chinese medicine (TCM), and validated with dry Houttuyniae Herba $(\mathrm{HH})$. In quantitative analysis, an SSDMC method involving nine components has been established with the desirable linearity $\left(r^{2} \geq 0.9998\right)$, precision (RSD $\left.<2.7 \%\right)$, accuracy $(97.4-103.1 \%)$ and ruggedness. Compared with the results obtained using the external standard method (ESM), this alternative SSDMC method was found to have no statistically significant differences. In fingerprint analysis, nine of fourteen peaks were identified. Simultaneously, $15 \mathrm{HH}$ samples from different origins were classified by similarity analysis and hierarchical clustering analysis (HCA). Additionally, the HPTLC method with three flavonoid markers was firstly established. The combination of SSDMC with the fingerprint and HPTLC method has been verified in the quality control of HH both quantitatively and qualitatively, and will provide a new quality evaluation pattern for TCMs.
\end{abstract}

Keywords Houttuyniae Herba, single standard to determine multi-components, fingerprint, high-performance thin layerchromatography

(Received January 5, 2015; Accepted March 10, 2015; Published June 10, 2015)

\section{Introduction}

Houttuyniae Herba $(\mathrm{HH})$, the terrestrial part of Houttuynia cordata Thunb. (Saururaceae), is a well-known traditional Chinese medicine (TCM) widely used in China and Japan, possessing a variety of pharmacological functions, including clearing heat, eliminating toxins, reducing swelling, discharging pus and relieving stagnation. ${ }^{1}$ Recently, it has also been demonstrated that it has good anti-bacterial, anti-viral, antiinflammatory, anti-tumor and immunomodulatory efficacy. ${ }^{2-10}$ Due to its clinical anti-bacterial efficacy, $\mathrm{HH}$ has been acknowledged as "broad-spectrum antibiotic in TCMs". ${ }^{5}$ It was also chosen as one of eight types of TCM that play a unique role in SARS. ${ }^{6}$ As the major component of various traditional Chinese medicinal preparations (TCMPs), including injection, tablet, oral preparation, eye drop, spray, etc., ${ }^{11-17}$ the quantity control of $\mathrm{HH}$ and its related TCMPs is of great importance to ensure their clinical efficacy.

In previous research, attention has mainly been focused on its volatile components, ${ }^{18}$ while no index of content determination or identification for non-volatile components has been recorded in Ch.P. 2010. ${ }^{1}$ Non-volatile components with high content and beneficial clinical actions partially consist of organic acids and flavonoids. ${ }^{19-20}$ Flavonoids, like rutin, hyperin, isoquercitrin,

$\dagger$ To whom correspondence should be addressed.

E-mail: lqyxm@hotmail.com quercitrin and quercetin, exhibit a wide range of biological effects, such as anti-inflammatory, anti-tumor, anti-virus, antibacteria and anti-oxidation. ${ }^{21-25}$ Organic acids, such as neochlorogenic acid, chlorogenic acid, cryptochlorogenic acid and caffeic acid, ${ }^{26}$ also have multiple actions including anticancer, anti-biotic, anti-viral and anti-hyperglycemic activity, etc. ${ }^{27}$ As a widely used TCM in clinics, this reveals an uncompleteness in $\mathrm{HH}$ quality evaluation. In conclusion, these factors make our research of considerable interest.

In an attempt to meet the quality evaluation requirements of TCMs, the conventional practice of selecting a single marker for testing has been gradually replaced by the determination of multiple active components. However, the limited availability of multiple reference substances, such as relatively large consumption of materials, time-consuming sample pretreatment, etc., hinders the wide popularization for any routine quality evaluation of TCMs. A concept of single standard to determine multi-components (SSDMC) was investigated quite intensively in recent years. ${ }^{28,29}$ With a view to finding an internal-standard substance, we attempted to use chlorogenic acid as the reference standard required in the SSDMC method. Consequently, chlorogenic acid served both as the external standard to determine the content of chlorogenic acid in $\mathrm{HH}$, and as the internal standard to simultaneously determine the other eight components according to the response factor (RF).

As a more comprehensive evaluation method, qualitative analysis should be a basic premise of quantitative analysis. The high-performance liquid chromatography (HPLC) fingerprint, 
which provides corresponding rich information concerning the chemical components contained in TCMs, along with highperformance thin layer-chromatography (HPTLC), which has been regarded as a visible, high-throughput, and cost-effective technology, was considered to be an effective way to identify TCMs. In this study, the HPTLC and HPLC fingerprint data established for $\mathrm{HH}$ were based on the principles of normalphase and reversed-phase chromatography, respectively. Additionally, both a similarity evaluation system and a hierarchical clustering analysis (HCA) were performed to classify samples from different origins according to the characteristic of common peaks in fingerprint chromatography.

Therefore, in order to evaluate the non-volatile components in $\mathrm{HH}$ both qualitatively and quantitatively, a combination method of SSDMC, fingerprint and HPTLC has already been applied. From a practical point of view, there will been an increasing awareness of the potential of using this combination method in the quality evaluation of $\mathrm{HH}$.

\section{Experimental}

\section{Instruments}

The Agilent 1260 HPLC system comprised a quaternary solvent delivery system, an on-line degasser, an autosampler, and a diode array detector coupled with an analytical workstation. System control and data analysis were processed with Agilent ChemStation software. The HPTLC method was performed on HPTLC plate silica gel $60(20 \times 10 \mathrm{~cm}$, Merck KGaA, Darmstadt, Germany).

\section{Reagents, chemicals and materials}

The HPLC-grade acetonitrile and methanol used for extraction were purchased from Fisher Scientific (Fisher Scientific, USA). Phosphoric acid, ethyl acetate, methanol, ethanol and formic acid were HPLC grade, and were obtained from Kemiou Chemical Reagent Co., Ltd. (Tianjin, China). Redistilled water was used for the preparation of a two-phase mobile solvent system.

Caffeic acid, rutin, hyperin, quercitrin and quercetin were purchased from the Institute for the Control of Pharmaceutical and Biological Products (Beijing, China). Neochlorogenic acid, chlorogenic acid, cryptochlorogenic acid and isoquercitrin were bought from Chengdu MUST Bio-technology Co., Ltd. (Sichuan, China). The purities of the nine compounds were all above $98 \%$.

Fifteen batches of dry $\mathrm{HH}$ purchased from 12 different provinces in China were collected. They were labeled as Hunan (No. 1, 2), Yunnan (No. 3), Zhejiang (No. 4), Anhui (No. 5), Sichuan (No. 6, 7), Henan (No. 8, 9), Jiangsu (No. 10), Guangdong (No. 11), Fujian (No. 12), Jiangxi (No. 13), Guizhou (No. 14) and Hubei (No. 15), respectively. All of the samples were identified by Prof. Ying Jia (Department of Traditional Chinese Medicine, Shenyang Pharmaceutical University, Liaoning, China).

\section{Preparation of sample solution and standard solution}

Preparation for SSDMC and fingerprint: After an amount of $500 \mathrm{mg}$ of finely powdered $\mathrm{HH}$ was accurately weighed and transfered to a $50-\mathrm{mL}$ glass-stoppered conical flask, $30 \mathrm{~mL}$ of $60 \%$ methanol was added. The solution was then sonicated for $30 \mathrm{~min}$. The extracts were filtered with a $0.22 \mu \mathrm{m}$ membrane filter. Preparation for HPTLC: $1.0 \mathrm{~g}$ of the sample was sonicated with $10 \mathrm{~mL}$ of $60 \%$ methanol for $30 \mathrm{~min}$.

A stock solution was prepared by dissolving the above- mentioned nine components (neochlorogenic acid, $4.58 \mathrm{mg}$; chlorogenic acid, $14.74 \mathrm{mg}$; cryptochlorogenic acid, $7.03 \mathrm{mg}$; caffeic acid, $2.58 \mathrm{mg}$; rutin, $5.29 \mathrm{mg}$; hyperin, $20.41 \mathrm{mg}$; isoquercitrin, $7.52 \mathrm{mg}$; quercitrin, $17.68 \mathrm{mg}$ and quercetin, $4.89 \mathrm{mg}$ ) in $10 \mathrm{~mL}$ of methanol, and stored at $4^{\circ} \mathrm{C}$.

After $0.2,0.4,0.8,2,4$ and $6 \mathrm{~mL}$ portions of the standard stock solution were quantitatively transfered to six $10-\mathrm{mL}$ amber volumetric flasks, they were diluted with methanol to volume, and mixed in order to construct regressive equations and to calculate the RFs and the relative retention times (RRTs).

\section{Chromatographic conditions of HPLC}

All analyses were separated on an Agilent Zorbax SB-C18 reversed-phase column $(4.6 \times 150 \mathrm{~mm}, 3.5 \mu \mathrm{m})$ coupled with an Agilent Zorbax Extend C18 guard column $(4.6 \times 10 \mathrm{~mm}$, $5 \mu \mathrm{m})$. The changeable detection wavelength was applied, and set at $327 \mathrm{~nm}$ in $0-14 \mathrm{~min}$ and $254 \mathrm{~nm}$ in $14-45 \mathrm{~min}$ for quantitative analysis. However, $254 \mathrm{~nm}$ was selected in the whole detection for qualitative fingerprint analysis. The flow rate and column temperature were maintained at $1.0 \mathrm{~mL} / \mathrm{min}$ and $20^{\circ} \mathrm{C}$, respectively. The mobile phase consisted of $0.05 \%$ phosphoric acid in water (A) and acetonitrile (B) with a linear gradient profile programed as follows for both quantitative analysis and qualitative assessment: $11 \%$ of the mobile phase (B) was chosen as the initial composition for $10 \mathrm{~min}$; $11-16 \%$ (B) for $2 \mathrm{~min}$; 16\% (B) for $12 \mathrm{~min} ; 16-25 \%$ (B) for $8 \mathrm{~min}$, and $25-44 \%$ for $13 \mathrm{~min}$ at last. Finally, $10 \mu \mathrm{L}$ of the sample solution was applied for injection.

\section{HPTLC conditions}

Test solutions $(5 \mu \mathrm{L})$ were applied to the HPTLC silica gel 60 plate with a band width of $1 \mathrm{~cm}$. The plate was then developed in a chamber containing a developing solvent system (ethyl acetate, methanol, formic acid $(15: 1: 2, \mathrm{v} / \mathrm{v} / \mathrm{v}))$ over a path of $8 \mathrm{~cm}$, sprayed with $1 \% \mathrm{AlCl}_{3}$ in ethanol, and finally examined under UV light at a wavelength of $365 \mathrm{~nm}$.

\section{Data analysis}

The RF and RRT of each analyte were calculated through a regression equation and obtained as the mean values calculated from the triplet of six gradient concentrations. $\mathrm{RF}_{\mathrm{i}}$ of the analytes was the ratio of the responses in a unit concentration between chlorogenic acid $\left(\mathrm{A}_{\mathrm{s}} / \mathrm{C}_{\mathrm{s}}\right)$ and the analyte $\left(\mathrm{A}_{\mathrm{i}} / \mathrm{C}_{\mathrm{i}}\right)$, where $\mathrm{A}$ and $\mathrm{C}$ were the peak area and the concentration obtained from the standard solution. ${ }^{30} \mathrm{RRT}_{\mathrm{i}}$ was calculated as the ratio of the retention time of the analyte $\left(t_{\mathrm{i}}\right)$ and chlorogenic acid $\left(t_{\mathrm{S}}\right)$.

\section{Evaluation methods}

Software named Similarity Evaluation System for Chromatographic Fingerprint of Traditional Chinese Medicine (Ver. 2009A) was employed to establish a reference chromatographic fingerprint and to calculate the similarity value of each sample. Furthermore, HCA was performed using SPSS 19.0 software.

\section{Results and Discussion}

\section{Optimization of the sample extraction conditions}

In order to obtain quantitative and complete extraction, the variables involved in the procedure were optimized. After comparing the peak areas of the nine investigated components, the optimal extraction method was demonstrated as that $i$ which finely powdered $\mathrm{HH}(0.5 \mathrm{~g})$ was sonicated with $30 \mathrm{~mL}$ of $60 \%$ $\mathrm{MeOH}$ for $30 \mathrm{~min}$. For a significant difference in the sensitivity 


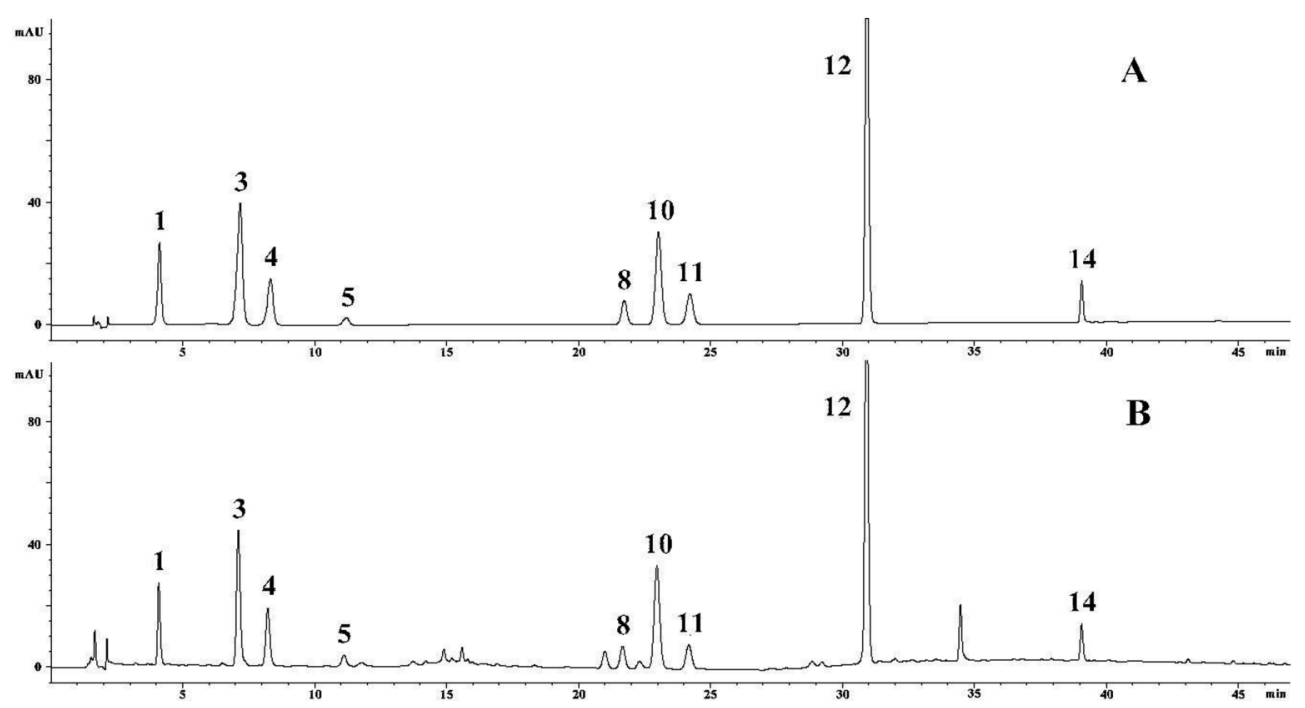

Fig. 1 Chromatograms of the quantitative analysis for Houttuyniae Herba. A. standard solution, B. sample solution. Nine components identified: 1. neochlorogenic acid, 3. chlorogenic acid, 4. cryptochlorogenic acid, 5. caffeic acid 8. rutin, 10. hyperin, 11. isoquercitrin, 12. quercitrin, 14. quercetin.

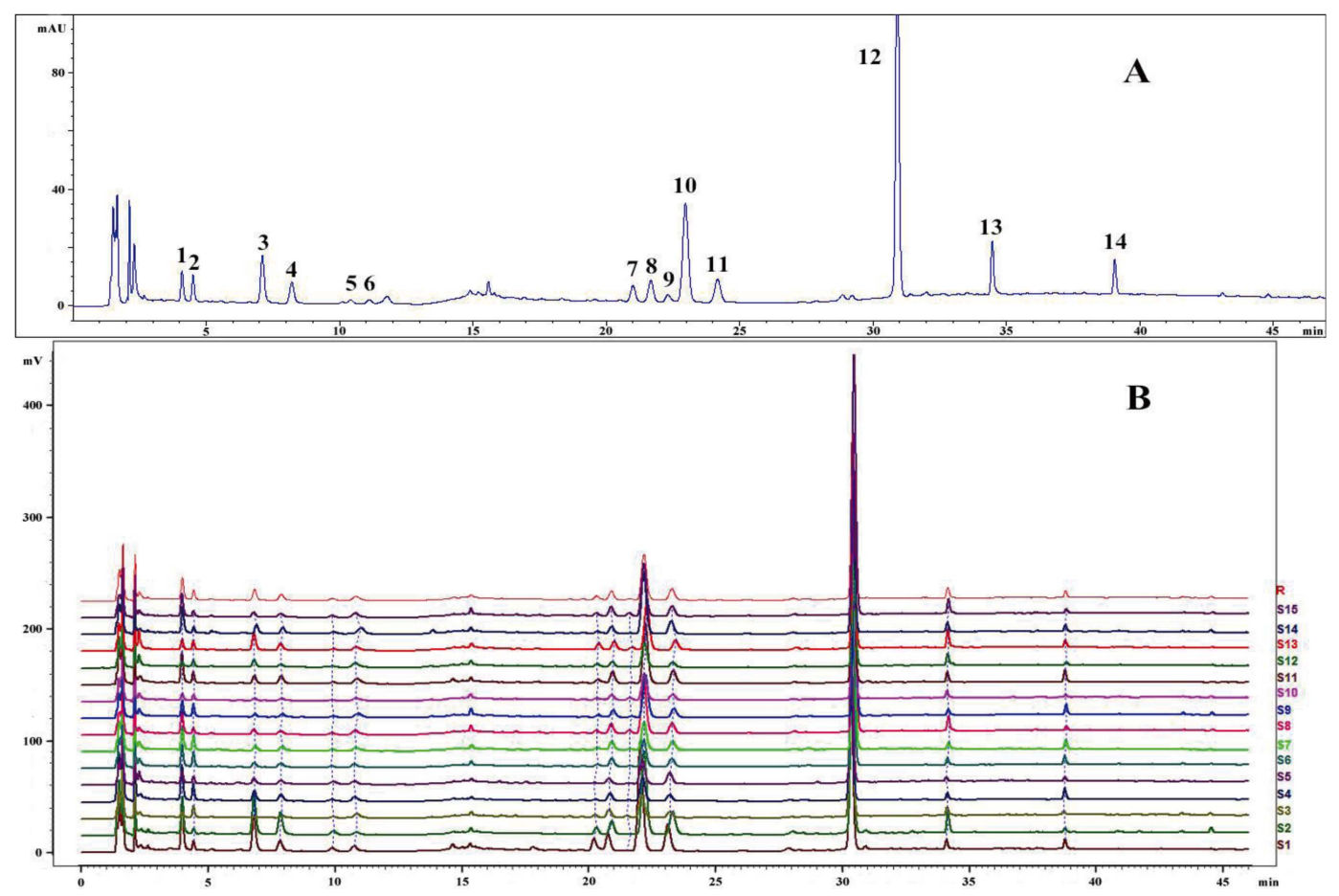

Fig. 2 Chromatograms of the HPLC fingerprint for Houttuyniae Herba. A. No. 13 sample, B. 15 batches of Houttuyniae Herba. Fourteen common peaks with 9 components identified: 1 . neochlorogenic acid, 3. chlorogenic acid, 4. cryptochlorogenic acid, 5. caffeic acid, 8. rutin, 10. hyperin, 11. isoquercitrin, 12. quercitrin, 14. quercetin.

between HPLC and HPTLC, $1.0 \mathrm{~g}$ of $\mathrm{HH}$ with $10 \mathrm{~mL} 60 \%$ methanol was employed in HPTLC extraction.

\section{Optimization of HPLC conditions}

To ensure a good resolution and an appropriate retention time of these quantitative compounds, the effects of several important factors were investigated in our previous work.

Three chromatographic columns were compared: Agilent
Zorbax SB-C18 $(4.6 \times 150 \mathrm{~mm}, 3.5 \mu \mathrm{m})$, GL Inertsil ODS-EP $(4.6 \times 150 \mathrm{~mm}, 5 \mu \mathrm{m})$, and Agilent Zorbax SB-C18 $(4.6 \times$ $250 \mathrm{~mm}, 5 \mu \mathrm{m})$. Of all the chromatographic columns, Agilent Zorbax SB-C18 $(4.6 \times 150 \mathrm{~mm}, 3.5 \mu \mathrm{m})$ was found to produce the best separation and a shortest retention time.

The wavelength for the detection of the nine compounds in the preparation was scanned over the entire UV range (200$400 \mathrm{~nm}$ ) to determine $\lambda_{\max }$. Flavonoids possessed the maximum 
absorption wavelength of $254 \mathrm{~nm}$, while organic acids showed a good absorption at $327 \mathrm{~nm}$, but a weak absorption at $254 \mathrm{~nm}$ for quantitative analysis. The achievement of the best responses for all of the target component resulted in a more serious requirement. An advanced technique, named the changeable detection wavelength, was applied to solve this problem. Thus, the detection wavelength was set at $327 \mathrm{~nm}$ in $0-14 \mathrm{~min}$ and $254 \mathrm{~nm}$ in $14-45 \mathrm{~min}$. However, a 3D chromatogram of the $\mathrm{HH}$ fingerprint showed more peaks at $254 \mathrm{~nm}$, which can fully reflect the component information in $\mathrm{HH}$. After a comprehensive comparison, $254 \mathrm{~nm}$ was selected as the best detection wavelength for the fingerprint.

The addition of phosphoric acid can improve the separation and peak symmetry of the components, especially for organic acids. The main reason remains that the appropriate amount of phosphoric acid can inhibit the ionization of phenolic acids.

On the basis of several trials, a step linear gradient was established for both of the HPLC quantitative analysis and the fingerprint of $\mathrm{HH}$. Additionally, it was found that good resolution and a symmetric peak shape were obtained when the column temperature was maintained at $20^{\circ} \mathrm{C}$, the flow rate was set at $1.0 \mathrm{~mL} / \mathrm{min}$ and the injection volume was $10 \mu \mathrm{L}$. Figure 1 shows the chromatographic profile of the $\mathrm{HH}$ sample solution A and the standard solution B obtained under the above-mentioned conditions. Figure 2 illustrates the best conditions of the fingerprint.

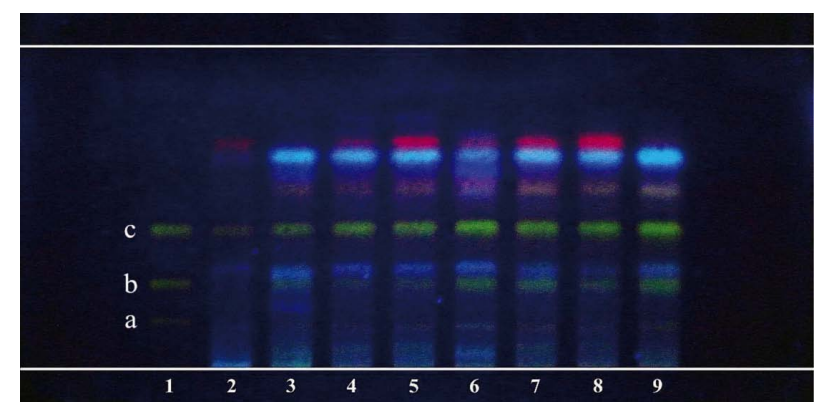

Fig. 3 HPTLC result of Houttuyniae Herba visualized under $365 \mathrm{~nm}$. Three components identified: a. rutin $(\mathrm{RF}=0.14)$, b. hyperoside $(\mathrm{RF}=0.25)$, c. quercitrin $(\mathrm{RF}=0.44), 1$. standard solution, 2 - 9 . sample solutions $\left(20^{\circ} \mathrm{C}, 40 \%\right)$ (HPTLC, Merck).

\section{Optimization of the HPTLC conditions}

Extensive trials were performed to achieve an optimal HPTLC method. The mixed solvent of acetate, methanol and formic acid was chosen as the developing solvent system. The addition of formic acid could help to prevent flavonoids from trailing, whereas the mass quantity of formic acid would move the components to the top of the chromatogram, leading to a badseparation. The results illustrated that plates developed by the solvent system of acetate-methanol-formic acid (15:1:2, v/v/v) over a path of $8 \mathrm{~cm}$ could produce the best separation (Fig. 3).

\section{Calculation of RFs and RRTs}

Chlorogenic acid was chosen as the internal standard for SSDMC and the fingerprint method because of its easy availability and good separation from the other components in the chromatogram. The RFs and RRTs were acquired by six concentration standard solutions. Triplicate experiments were performed, and the average RFs and RRTs were calculated shown in Table 1. The results proved that the RFs and RRTs were stable for different as standard solutions.

\section{Validation of SSDMC method}

The linearity equation was constructed based on six concentration levels. All of the analytes showed excellent linear behaviors over the investigated concentration ranges $\left(r^{2} \geq\right.$ 0.9998). The limit of detection (LOD) and limit of quantitation (LOQ) of the nine analytes were $0.04-0.08$ and $0.12-$ $0.28 \mu \mathrm{g} / \mathrm{mL}$. Detailed descriptions of the regression equations, LOD and LOQ are presented in Table 1.

The precision was measured based on the intra-day and interday variability. The relative standard deviations (RSDs) of the content of the nine markers obtained by the external standard method (ESM) and SSDMC methods were in the range of $0.4-$ $2.7 \%$.

The accuracy was measured by the recovery test. The recoveries of the nine investigated compounds ranged from 97.4 to $103.1 \%$, with $\mathrm{RSD}<2.2 \%$.

These results showed that the precision and accuracy of the alternative method would suffice for the routine quality control requirements of $\mathrm{HH}$.

The ruggedness of RF and RRT of the nine components were compared with two different equipments (Agilent 1260 and Agilent 1100) and three columns (Agilent Zorbax SB-C18 $(4.6 \times 150 \mathrm{~mm}, 3.5 \mu \mathrm{m})$, GL Inertsil ODS-EP $(4.6 \times 150 \mathrm{~mm}$, $5 \mu \mathrm{m})$, Agilent Zorbax SB-C18 $(4.6 \times 250 \mathrm{~mm}, 5 \mu \mathrm{m}))$ through six concentration standard solutions. It exhibited that RRT has great differences in the columns, with the maximum RSD of

Table 1 Regressive equations, correlation coefficients, linearity ranges, LOD, LOQ, RF and RRT for SSDMC

\begin{tabular}{|c|c|c|c|c|c|c|c|}
\hline Analyte & Regressive equation & $\begin{array}{c}\text { Linear range/ } \\
\mu \mathrm{g} \mathrm{mL}^{-1}\end{array}$ & $r^{2}$ & $\begin{array}{c}\text { LOD/ } \\
\text { ng mL }^{-1}\end{array}$ & $\begin{array}{c}\mathrm{LOQ/} \\
\mathrm{ng} \mathrm{mL}^{-1}\end{array}$ & RF & RRT \\
\hline 1 & $y=24.47 x-3.849$ & $1.301-41.62$ & 0.9999 & 44.98 & 149.9 & 1.023 & 0.574 \\
\hline 2 & $y=28.42 x-10.42$ & $2.948-94.34$ & 0.9999 & 71.40 & 238.0 & 1.000 & 1.000 \\
\hline 3 & $y=27.76 x-8.487$ & $1.294-41.39$ & 0.9998 & 80.66 & 268.9 & 1.050 & 1.162 \\
\hline 4 & $y=20.66 x-1.503$ & $0.387-12.38$ & 0.9999 & 79.26 & 264.2 & 1.400 & 1.560 \\
\hline 5 & $y=20.24 x-0.9841$ & $0.804-25.73$ & 1.0000 & 68.93 & 229.8 & 1.382 & 3.024 \\
\hline 6 & $y=26.80 x-1.916$ & $2.530-80.99$ & 1.0000 & 76.66 & 255.5 & 1.032 & 3.208 \\
\hline 7 & $y=27.02 x-1.383$ & $0.902-28.87$ & 1.0000 & 82.81 & 276.0 & 1.037 & 3.377 \\
\hline 8 & $y=28.73 x+2.258$ & $6.368-203.8$ & 1.0000 & 44.14 & 147.1 & 0.954 & 4.303 \\
\hline 9 & $y=19.41 x-1.494$ & $0.734-23.47$ & 1.0000 & 34.57 & 115.2 & 1.453 & 5.431 \\
\hline
\end{tabular}

1. Neochlorogenic acid, 2. Chlorogenic acid, 3. Cryptochlorogenic acid, 4. Caffeic acid, 5. Rutin, 6. Hyperin, 7. Isoquercitrin, 8. Quercitrin,

9. Quercetin. 
Table 2 Content and similarity of 15 batches of Houttuyniae Herba samples

\begin{tabular}{|c|c|c|c|c|c|c|c|c|c|c|c|c|c|}
\hline \multirow{3}{*}{$\begin{array}{c}\text { No. } \\
1\end{array}$} & \multicolumn{9}{|c|}{ Content by SSDMC, \% } & \multicolumn{2}{|c|}{ Total content, $\%$} & \multirow{2}{*}{\multicolumn{2}{|c|}{$\begin{array}{c}\text { RSD, } \% \\
\text { Similarity }\end{array}$}} \\
\hline & 1 & 2 & $3(\mathrm{ESM})$ & 4 & 5 & 6 & 7 & 8 & 9 & ESM & SSDMC & & \\
\hline & 0.2090 & 0.1937 & 0.0661 & 0.0222 & 0.0593 & 0.3215 & 0.0826 & 0.3364 & 0.0205 & 1.3203 & 1.3114 & 0.5 & 0.937 \\
\hline 2 & 0.1511 & 0.2299 & 0.1443 & 0.0096 & 0.0480 & 0.2461 & 0.0740 & 0.3693 & 0.0098 & 1.2901 & 1.2820 & 0.5 & 0.975 \\
\hline 3 & 0.0192 & 0.0087 & 0.0044 & 0.0158 & 0.0286 & 0.0668 & 0.0194 & 0.3535 & 0.0084 & 0.5259 & 0.5248 & 0.2 & 0.973 \\
\hline 4 & 0.1429 & 0.0554 & 0.0356 & 0.0330 & 0.0277 & 0.0676 & 0.0196 & 0.2271 & 0.0227 & 0.6350 & 0.6316 & 0.4 & 0.985 \\
\hline 5 & 0.0501 & 0.0324 & 0.0214 & 0.0175 & 0.0191 & 0.0700 & 0.0348 & 0.2161 & 0.0085 & 0.4715 & 0.4698 & 0.3 & 0.994 \\
\hline 6 & 0.0874 & 0.0259 & 0.0227 & 0.0203 & 0.0275 & 0.0776 & 0.0254 & 0.2708 & 0.0181 & 0.5778 & 0.5757 & 0.3 & 0.991 \\
\hline 7 & 0.0897 & 0.0270 & 0.0220 & 0.0224 & 0.0291 & 0.0811 & 0.0272 & 0.2936 & 0.0195 & 0.6138 & 0.6117 & 0.3 & 0.990 \\
\hline 8 & 0.0913 & 0.0282 & 0.0200 & 0.0143 & 0.0336 & 0.1553 & 0.0341 & 0.4786 & 0.0092 & 0.8676 & 0.8647 & 0.3 & 0.993 \\
\hline 9 & 0.0602 & 0.0182 & 0.0164 & 0.0217 & 0.0252 & 0.1259 & 0.0283 & 0.3011 & 0.0208 & 0.6199 & 0.6179 & 0.3 & 0.998 \\
\hline 10 & 0.0248 & 0.0128 & 0.0112 & 0.0098 & 0.0181 & 0.0747 & 0.0182 & 0.2429 & 0.0060 & 0.4197 & 0.4185 & 0.2 & 0.991 \\
\hline 11 & 0.1200 & 0.0437 & 0.0491 & 0.0319 & 0.0428 & 0.1712 & 0.0417 & 0.3662 & 0.0257 & 0.8957 & 0.8924 & 0.3 & 0.999 \\
\hline 12 & 0.0192 & 0.0400 & 0.0171 & 0.0163 & 0.0188 & 0.0707 & 0.0149 & 0.1911 & 0.0075 & 0.3975 & 0.3957 & 0.4 & 0.995 \\
\hline 13 & 0.0366 & 0.0943 & 0.0422 & 0.0184 & 0.0308 & 0.1401 & 0.0297 & 0.2631 & 0.0148 & 0.6736 & 0.6700 & 0.4 & 0.994 \\
\hline 14 & 0.1080 & 0.0449 & 0.0293 & 0.0222 & 0.0246 & 0.1742 & 0.0394 & 0.3656 & 0.0135 & 0.8251 & 0.8216 & 0.3 & 0.998 \\
\hline 15 & 0.0467 & 0.0712 & 0.0412 & 0.0155 & 0.0265 & 0.1243 & 0.0264 & 0.2514 & 0.0121 & 0.6183 & 0.6153 & 0.4 & 0.998 \\
\hline
\end{tabular}

1. Neochlorogenic acid, 2. Chlorogenic acid, 3. Cryptochlorogenic acid, 4. Caffeic acid, 5. Rutin, 6. Hyperin, 7. Isoquercitrin, 8. Quercitrin,

9. Quercetin.

$12.5 \%$, while it was fluctuated in a relative narrow range with different equipments $(\mathrm{RSD}<4.6 \%)$. It was conjectured that columns with different packing or length would affect the retention behavior of various compounds. In contrast, RF was relatively stable both in different columns and equipments, especially for neochlorogenic acid $(1.0 \%)$

The ruggedness of the established method was also evaluated by examining its stability with small variations of the procedural parameters, including the acid concentration in the mobile phase $\left( \pm 0.025 \% \quad \mathrm{H}_{3} \mathrm{PO}_{4}\right)$, the ratio of the mobile phase $( \pm 1 \%)$, programs of the mobile phase $( \pm 1 \mathrm{~min})$, the wavelength $( \pm 3 \mathrm{~nm})$, the flow rate $( \pm 0.1 \mathrm{~mL} / \mathrm{min})$, the injection volume $( \pm 2 \mu \mathrm{L})$, and the column temperature $\left( \pm 5^{\circ} \mathrm{C}\right)$. It was proved that a slight variation of the wavelength had a great influence on the RFs of the nine components (RSD $>3 \%$ ), especially for quercitrin $(\mathrm{RSD}=4.7 \%)$. The RRTs of flavonoids were quite variably associated with different flow rates, the ratio of the mobile phase and programs of the mobile phase. Besides, even a slight change of mobile phase would have a significant effect on the content determination result of rutin $(\mathrm{RSD}=10.9 \%$ ). Other factors had slight influences on the RFs, RRTs and the content of the components. The fluctuation was proportionable (RSD $<3.0 \%)$. The results showed that the RRTs of flavonoids were highly sensitive to the variation of the flow rate, the ratio of the mobile phase and the programs of the mobile phase for the reason that the chromatography behavior of organic acids and flavonoids were different under these conditions. The influence on the RFs along with the change of the wavelength was significant, mainly because the response variations of organic acids and flavonoids affected by different detection wavelengths were disproportionate. Besides, there was a peak possessing a similar retention time with rutin; the resolution of these two adjacent peaks was very sensitive to the ratio of the mobile phase, which resulted in changes in the content of rutin.

Therefore, it was recommended that the flow rate, wavelength and programs of the mobile phase should not be changed very much, and that the ratio of mobile phase should be controlled strictly. The $\mathrm{pH}$ of the mobile phase, the injection volume and the column temperature can be slightly adjusted to meet the system suitability.

\section{Validation of identification methods for qualitative analysis}

The HPLC fingerprint and the HPTLC method were respectively established by using reverse-phase and normalphase chromatography, as discussed in this section. Details on qualitative analysis are discussed in later sections.

\section{HPLC fingerprint method}

The method was validated, including the precision, reproducibility and stability test, by the analysis of sample solutions of No. 13. All of the RSDs obtained from the validation test were within the range of $2.0-4.1 \%$ for relative peak area (RPA) and $0.06-1.6 \%$ for RRT, which indicated the feasibility of the method and the stability of the sample solution within $24 \mathrm{~h}$

\section{HPTLC method}

In this study, the HPTLC method was validated based on the specificity, stability and ruggedness. It proved that the sample solutions were stable within 3 days, and that a slight change of temperature $\left(10-30^{\circ} \mathrm{C}\right)$ and humidity $(30-90 \%)$ had no remarkable influence on the chromatograms of the sample and standard solutions. However, the bands on the HPTLC plate were narrower and easy to distinguish, but were not clear and acceptable on the TLC plate. Consequently, the best chromatography was conducted by HPTLC plates (Merck), at a low temperature of $20^{\circ} \mathrm{C}$ and a moderate humidity of $40 \%$.

\section{HPLC quantitative analysis}

To validate the SSDMC and ESM method, 15 batches of dry HH samples from different habitats were analyzed. The amounts of the 9 investigated components were calculated (Table 2). There were no significant differences observed between these two methods (SSDMC and ESM) using the $t$-test ( $p=5.49$, $n=15)$.

\section{Similarity analysis and HCA of the HPLC fingerprint}

A fingerprint, especially a high-resolution chromatography, must have a sufficient preparation of representative samples of authenticity. Therefore, we analyzed the previously collected 15 batches of samples from different origins by the optimized extraction method and the HPLC method. The HPLC fingerprint 


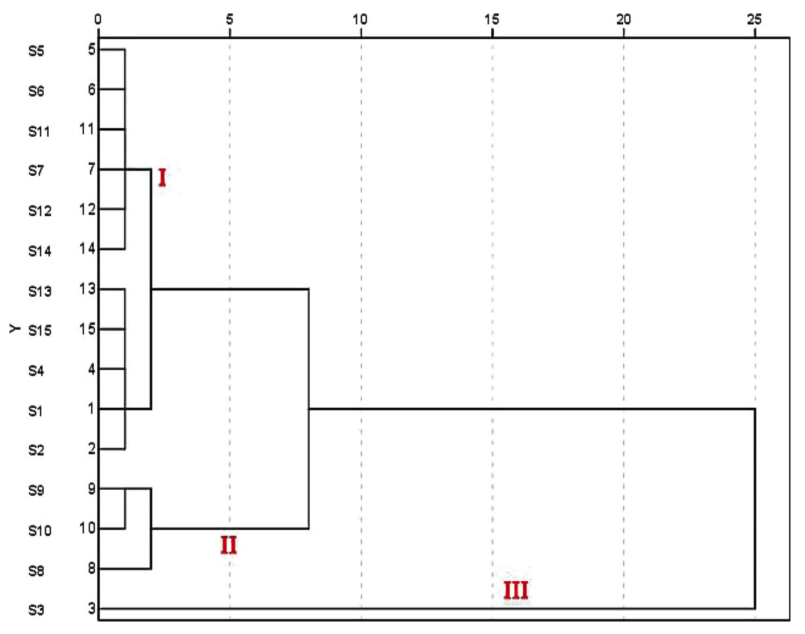

Fig. 4 Dendrogram of clustering of 15 batches of Houttuyniae Herba samples.

common pattern of $\mathrm{HH}$ was established by the software of the Similarity Evaluation System for Chromatographic Fingerprint of Traditional Chinese Medicine (Ver. 2009A). With the No. 13 sample as the reference sample, the standard reference chromatography of the HPLC fingerprint was generated by applying the median method from a general comparison of all samples. Figure $2 \mathrm{~B}$ illustrates that the chromatograms of $\mathrm{HH}$ contained 14 common peaks within 45 min. Among them, nine component peaks were identified by comparing the retention times and the UV spectra with the reference standards, including neochlorogenic acid (1), chlorogenic acid (3), cryptochlorogenic acid (4), caffeic acid (6), rutin (8), hyperin (10), isoquercitrin (11), quercitrin (12) and quercetin (14). The chlorogenic acid (3) was selected as the inter-reference standard to calculate RPAs and RRTs of the other common characteristic peaks. The similarity values of all of the samples to the reference fingerprint were more than 0.937 , as given in Table 2 . The results indicated that it is easy to identify $\mathrm{HH}$ based on this chromatographic fingerprint.

HCA was operated using SPSS 19.0 software according to RPA of all the samples; the results are displayed in Fig. 4. Fifteen samples of $\mathrm{HH}$ were divided into three main clusters (I, II and III). S8, S9 from Henan province and S10 from Jiangsu province were classified in cluster II. Only S3 from Yunnan province was in cluster III, while the others belonged to cluster I. The most crucial factor leading to this result may be the cultivation conditions. The growth of $\mathrm{HH}$ requires a suitable level of temperature $\left(20-25^{\circ} \mathrm{C}\right)$ and the soil relative humidity $(80-90 \%)$. Without considering such factors as the soil nutrient supply, the humidity has become the most important factor that affects the content of nutrients in $\mathrm{HH} .{ }^{31}$ Consequently, when taking into account the climatic conditions of these provinces, it was obvious that samples in cluster I were grown in subtropical extremely-humid or humid regions, while samples in cluster II were grown in sub-humid warm temperate regions. Yunnan province has two seasons, namely the wet and dry seasons, and the precipitation is not uniformly distributed in the two seasons that can lead to differences in the growth of $\mathrm{HH}$, which may be the reason for cluster III of its own.

\section{HPTLC qualitative analysis}

Fifteen batches of $\mathrm{HH}$ were identified according to the validated method. Similar chromatograms were shown for all sample solutions under UV light $(365 \mathrm{~nm})$, shown in Fig. 3. Three yellow bands due to rutin, hyperin and quercitrin from the lower to upper parts of the plate were found in these chromatograms; the corresponding $\mathrm{RF}$ values were approximately $0.14,0.25$ and 0.44 , respectively. The chromatograms of the sample solution also exhibited several yellowish-white, orange, blueish-white and red bands at the same positions.

\section{Conclusions}

This study reports on the applicability of comprehensive analytical techniques, the experimental procedures and the study findings. Three methods were involved with improvement and innovation. The HPLC was applied to establish the fingerprint and the SSDMC method, which offered us a possible way to simultaneously evaluate the quality of $\mathrm{HH}$ both quantitatively and qualitatively. The HPLC method provided correspondingly rich component information, while the traditional HPTLC method with three makers of flavonoids showed simpler, more economic and more fingerprint resistance advantages; it was reported in a quality assessment of $\mathrm{HH}$ for the first time. Compared with the existing quality assessment of $\mathrm{HH},{ }^{1}$ the simple and rapid combination method of the fingerprint, HPTLC and SSDMC method made the quality evaluation results more intuitive, reliable and comprehensive. It also provided a scientific basis for improving its quality standard.

\section{Acknowledgements}

This study was financially supported by Liaoning Innovative Research Team in University (LNIRT, Grant No. LT2013022).

\section{References}

1. National Commission of Chinese Pharmacopoeia, Pharmacopoeia of People's Republic of China, 2010, Chemical Industry Press, Beijing, 208.

2. W. Zhang, S. Y. Pan, and F. G. Lu, Pract. Prev. Med., 2008, 15,312 .

3. L. C. Chiang, J. S. Chang, C. C. Chen, L. T. Ng, and C. C. Lin, Am. J. Chin. Med., 2003, 31, 355.

4. K. Hayashi, M. Kamiya, and T. Hayashi, Planta Med., 1995, 61, 237.

5. X. Q. Du, M. Y. Chen, and Y. Xu, Jiangxi Journal of Traditional Chinese Medicine, 2012, 2, 66.

6. H. M. Lu, Y. Z. Liang, L. Z. Yi, and X. J. Wu, J. Ethnopharmacol., 2006, 104, 245.

7. I. S. Kim, J. H. Kim, J. S. Kim, C. Y. Yun, D. H. Kim, and J. S. Lee, J. Ethnopharmacol., 2007, 112, 90.

8. S. K. Kim, S. Y. Ryu, J. No, S. U. Choi, and Y. S. Kim, Arch. Pharmacol. Res., 2001, 24, 518.

9. G. Z. Li, O. H. Chai, M. S. Lee, E. H. Han, H. T. Kim, and C. H. Song, Biol. Pharm. Bull., 2005, 28, 1864.

10. K. M. Lau, K. M. Lee, and C. M. Koon, J. Ethnopharmacol., 2008, 118, 79 .

11. L. D. Li, J. Y. Zhang, P. He, W. W. Sun, and Y. K. Li, Sci. Techonol. Rev. (Beijing, China), 2010, 28, 36.

12. J. W. Qin and Z. G. Song, China's Naturopathy, 2011, 19, 47.

13. Z. Q. Wu, Straits Science, 2004, 5, 36.

14. S. Y. Zhu, China Pharmacist, 2002, 5, 600. 
15. L. Yang, China Pharmaceuticals, 2006, 19, 47.

16. J. Tang, W. J. Fang, C. Y. Shi, and W. Q. Wang, Chinese Journal of Hospital Pharmacy, 2008, 28, 1255.

17. T. Xia, J. Q. Chen, J. Y. Guo, C. B. Yao, and L. F. Lin, China Health Care \& Nutrition, 2013, 1, 479.

18. R. X. Hu, B. M. Xiao, Z. J. Tan, W. N. Zhao, B. Y. Xie, D. P. Xie, and C. R. Wu, China Pharmaceuticals, 2008, 17, 23.

19. J. Meng, Y. S. Zhou, Z. Z. Zhao, and X. P. Dong, Journal of China Pharmaceutical University, 2007, 38, 516.

20. Q. C. Peng, Z. N. Yang, J. W. Hu, W. S. Chen, and C. X. Li, Journal of Jiangxi Normal University (Natural Sciences Edition). 2008, 32, 645.

21. Z. H. Zhang, L. P. Cao, and L. Zhong, Herald of Medicine, 2007, 26, 758 .

22. K. Huang, X. B. Yang, and Z. M. Huang, Herald of Medicine, 2009, 28, 1046.

23. X. X. Zhu, F. S. Jiang, and Z. S. Ding, Journal of Snake, 2012, 24, 47.
24. H. W. Fan, W. Qu, Y. Li, and M. Sun, Chinese Journal of Hospital Pharmacy, 2008, 28, 528.

25. J. He, R. He, and F. W. Yan, Food Sci. Technol., 2014, 39, 198.

26. S. Y. Jang, J. S. Bae, Y. H. Lee, K. Y. Oh, K. H. Park, and Y. S. Bae, Nat. Prod. Res., 2011, 25, 222.

27. W. H. Wu, Z. Kang, D. S. Ouyang, and H. H. Zhou, Nat. Prod. Res. Dev., 2006, 18, 691.

28. H. L. Liao, Q. Li, R. Liu, J. J. Liu, and K. S. Bi, Anal. Sci., 2014, 30, 1157.

29. Y. Y. Du, Q. Li, J. J. Liu, Y. D. Yin, and K. S. Bi, Anal. Methods, 2014, 6, 5891.

30. J. J. Hou, W. Y. Wu, J. Da, S. Yao, H. L. Long, Z. Yang, L. Y. Cai, M. Yang, X. Liu, B. H. Jiang, and D. A. Guo, J. Chromatogr. A, 2011, 1218, 5618.

31. X. J. Wu, S. J. Jiang, M. Z. Hu, L. Wei, X. H. Jiang, and J. Zhang, Journal of Anhui Agricultural Sciences, 2007, 35, 742 . 